\title{
Hérnia diafragmática oculta após trauma - Relato de caso
}

\author{
Hidden post-traumatic diaphragmatic hernia - Case Report \\ Hernia diafragmática oculta post-traumática - Relato de un caso
}

Geovane Biase Gomes da Costa ${ }^{1 *}$, Ana Maria Sampaio de Melo², Valéria Lopes de Andrade ${ }^{2}$, José João Corrêa Bergamasco ${ }^{1}$, Juan Eduardo Rios Rodriguez ${ }^{3}$, Brígida Thaine Fernandes Cabral ${ }^{3}$.

\section{RESUMO}

Objetivo: Relatar um caso de hérnia diafragmática pós-traumática de diagnóstico tardio com sintomas discretos e discutir sobre as bases do diagnóstico e manejo atuais das mesmas. Relato do caso: A paciente do sexo feminino, 46 anos, deu entrada em serviço de urgência na cidade de Manaus, com queixa de dispneia discreta, dor torácica esquerda e dor abdominal mais localizada em hipocôndrio esquerdo, com piora dos sintomas há dois dias e história pregressa de trauma contuso. Realizada tomografia computadorizada com evidência de hérnia volumosa em hemitórax esquerdo, que não condizia com os sintomas ou o estado geral da paciente. Foi realizada laparotomia exploradora com incisão mediana infra umbilical. Visualizou-se lesão de aproximadamente 16 centímetros em diafragma seguida de correção cirúrgica das lesões. Considerações Finais: As lesões diafragmáticas podem ser diagnosticadas imediatamente, em meses ou anos após o trauma. Este relato ilustra mais um caso raro de hérnia diafragmática traumática pouco sintomática em contraste com o grande volume de alças intestinais em cavidade torácica.

Palavras-chave: Diafragma, Ferimentos e Lesões, Traumatismos Torácicos, Cirurgia Geral.

\begin{abstract}
Objective: To report a case of post-traumatic diaphragmatic hernia of late diagnosis with discrete symptoms and to discuss the basis of current diagnostic and management of these. Case report: A 46-year-old female patient was admitted to the emergency department of the city of Manaus, complaining of mild dyspnea, left chest pain, and abdominal pain more localized in the left hypochondrium, worsening symptoms two days ago. history of blunt trauma. Computed tomography was performed for evaluation, with evidence of bulky hernia in the left hemithorax, which did not match the symptoms or the general condition of the patient. An exploratory laparotomy above and below the umbilicus medial incision was performed. A lesion of approximately $16 \mathrm{~cm}$ in diaphragm was found with surgical correction of the lesions in sequence. Final Considerations: Diaphragmatic lesions can be diagnosed immediately, months or years after the trauma. This report illustrates another rare case of poorly symptomatic traumatic diaphragmatic hernia in contrast to the large volume of intestinal loops in the thoracic cavity.
\end{abstract}

Keywords: Diaphragm, Wounds and Injuries, Thoracic Injuries, General Surgery.

\footnotetext{
${ }^{1}$ Hospital Universitário Getúlio Vargas. *E-mail: geovane_biase@hotmail.com

2 Instituto de Cirurgia do Estado do Amazonas.

${ }^{3}$ Universidade Federal do Amazonas.
} 


\section{RESUMEN}

Objetivo: Informar de un caso de hernia diafragmática post-traumática de diagnóstico tardío con síntomas discretos y discutir sobre las bases del diagnóstico y manejo actuales de las mismas. Relato del caso: Paciente del sexo femenino, de 46 años, entró en servicio de urgencia en la ciudad de Manaus, con queja de dispnea discreta, dolor torácico izquierdo y dolor abdominal más localizado en hipocondrio izquierdo, con empeoramiento de los síntomas hace dos días y historia previa de trauma contuso. Se realizó una tomografía computarizada con evidencia de hernia voluminosa en hemitórax izquierdo, que no concuerda con los síntomas o el estado general de la paciente. Se realizó laparotomía exploradora con incisión mediana infra e supraumbilical. Se observó lesión de aproximadamente 16 centímetros en diafragma seguida de corrección quirúrgica de las lesiones. Considerações finais: As lesões diafragmáticas podem ser diagnosticadas imediatamente, em meses ou anos após o trauma. Este relato ilustra mais um caso raro de hérnia diafragmática traumática pouco sintomática em contraste com o grande volume de alças intestinais em cavidade torácica.

Palavras-clave: Diafragma, Heridas e Lesiones, Traumatismos Torácicos, Cirugía General.

\section{INTRODUÇÃO}

A hérnia diafragmática é uma ruptura do músculo diafragma, que causa uma conexão entre a cavidade torácica e a abdominal. Essa hérnia tem um padrão fisiológico diferente de outros tipos, como os da parede abdominal, já que apresenta dois vetores de força: a pressão positiva exercida pela cavidade abdominal e a pressão negativa da cavidade torácica que realiza uma função succtora nas alças e outros órgãos (ERCAN M, et al., 2016)

A hérnia diafragmática é de ocorrência rara, envolvendo duas cavidades corporais grandes, ou seja, pode causar repercussões sistêmicas variadas e complicações diversas. A sua classificação pela etiologia é dividida em congênita e traumática, sendo a primeira um evento mais comum e presente principalmente em pacientes pediátricos, com pouquíssimos casos relatados na população adulta (ČALA Z, et al., 2017). Em situações de urgência e emergência, a lesão do diafragma se apresenta de maneira constante em lesões por arma de fogo ou branca, tanto de orifício de entrada abdominal ou torácico (13-19\%), enquanto lesões contusas como acidentes automobilísticos e quedas de grande altura apresentam uma frequência menor no aparecimento das mesmas, não chegando a mais de 5\% (JÚNIOR GAP, 2000). No entanto, pesquisas mais recentes sugerem mudanças no padrão de diagnóstico de hérnias traumáticas do diafragma, relacionando trauma fechado de abdômen como principal causa (ABDELSHAFY M e KHALIFA YSE, 2018).

Seu diagnóstico quando precoce (como pouco tempo após o trauma) é realizado durante a laparotomia de exploração cirúrgica, ou tardio quando apresenta sintomas respiratórios ou de obstrução do trato intestinal. Para avaliação, exames complementares como Tomografia Computadorizada ou radiografia são essenciais, principalmente em situações crônicas ou complicados (ZHAO H, et al., 2017). É preciso ter cuidado com o paciente em fase aguda, já que não apresenta sintomas claros ou muito exacerbados na maioria dos casos, podendo evoluir para encarceramento (fase latente) e estrangulamento (fase crônica). O descuido com o paciente pode levar a complicações durante procedimentos, como em tentativas de drenagem de tórax pela suspeita de hemotórax ou quilo tórax que acarretam lesão em vísceras ocas durante a inserção do tubo de drenagem. (BLITZ M e LOUIE BE, 2009)

O tamanho da hérnia não apresenta classificação definitiva, apenas a American Association for the Surgery of Trauma (AAST) classificou o tamanho lesão no tecido diafragmático em 5 níveis, de contusão até lesões maiores que 25 centímetros com perda de tecido orgânico (MOORE EE, et al., 1995). No entanto, a clínica não necessariamente está relacionada com o tamanho da herniação, e a lesão pode vir a aumentar com o decorrer da evolução natural, já que as pressões abdominal e intratorácica atuam de modo que a tendência é o aumento da extensão da ferida. (ALLAN Z, et al., 2017). A seguir, apresentamos um caso de difícil conduta, devido a falta de relação da clínica inicial com a condição física da paciente. 


\section{DETALHAMENTO DO CASO}

Mulher de 46 anos, natural e procedente do interior do Amazonas foi admitida em unidade hospitalar de Pronto-socorro na cidade de Manaus/AM com queixa principal de dispneia, com progressão de sintomas há dois dias. A mesma nega que a dispneia esteja relacionada a esforços, e não apresentou secreção purulenta ou sanguinolenta, com presença de tosse intermitente, mas não relevante para a própria paciente. Referia dor discreta em base de hemitórax esquerdo (HE) e em abdômen superior, mais localizado em hipocôndrio esquerdo. Negou comorbidades ou alergias, mas relata história pregressa de acidente automobilístico motocarro em que a mesma estava como passageira de moto com capacete e sofreu trauma contuso em $\mathrm{HE}$ e em abdômen há 2 meses. Nega etilismo, tabagismo ou uso de drogas ilícitas.

Ao exame físico (apesar de não ser atendimento inicial ao trauma, houve a decisão de seguir um padrão de atendimento ao trauma básico): paciente estava em bom estado geral, lúcida e orientada em tempo e espaço, dispneica, normocorada, anictérica e acianótica. Em ausculta pulmonar apresentava murmúrio vesicular abolido em terço superior-médio até inferior de HE e ruídos hidroaéreos audíveis em terço inferior, expansibilidade torácica maior em hemitórax direito. Ausculta cárdica sem alterações relevantes, frequência cárdica de 89 bpm, PA de 137/86 mmHg. Abdômen apresentava-se globoso, sem abaulamentos importantes, sem cicatriz cirúrgica, discretamente doloroso a palpação superficial, com piora da dor ao aprofundamento, no entanto não apresenta irritação peritoneal em nenhum quadrante, ou qualquer sinal irritativo, como Murphy ou Gueneau de Mussy.

Paciente possuía radiografia prévia realizada em cidade próxima, apresentando aumento de nível da bulha gástrica, mas devido a qualidade da mesma, uma tomografia computadorizada com topograma foi realizada para elucidação diagnóstica, com suspeita inicial de hemotórax ou hérnia diafragmática, sendo esta a razão por qual a drenagem torácica precoce não fora realizada. Em exame, evidenciou-se hérnia volumosa de alças intestinais em cavidade torácica esquerda, com desvio de mediastino para a direita (Figuras 1 e 2).

Figura 1. Tomografia computadorizada em corte transversal evidenciando conteúdo abdominal em hemitórax esquerdo.

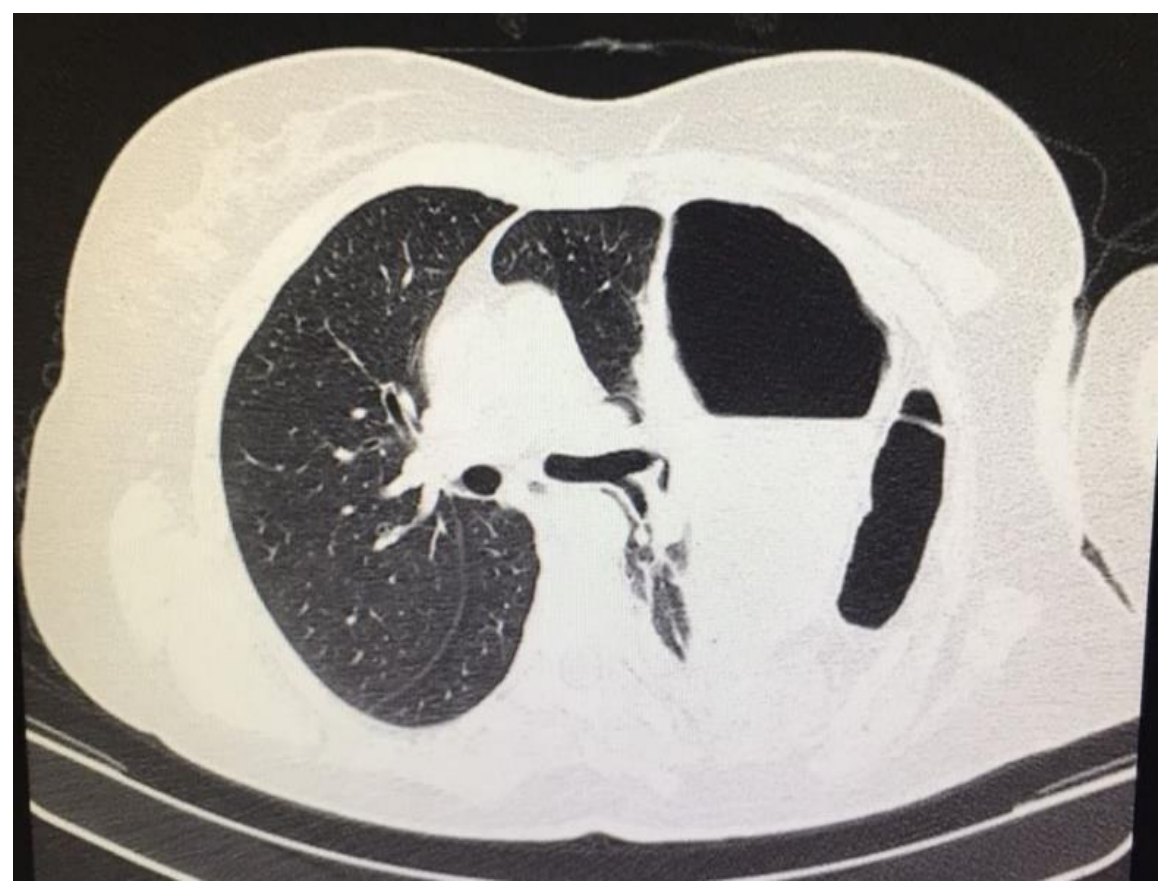

Fonte: Dados da pesquisa, 2018. 
Figura 2. Topograma realizado durante TC, note-se o desvio do mediastino para direita, com compressão de parênquima pulmonar direito, devido a presença de conteúdo abdominal em grande quantidade em hemitórax contralateral.

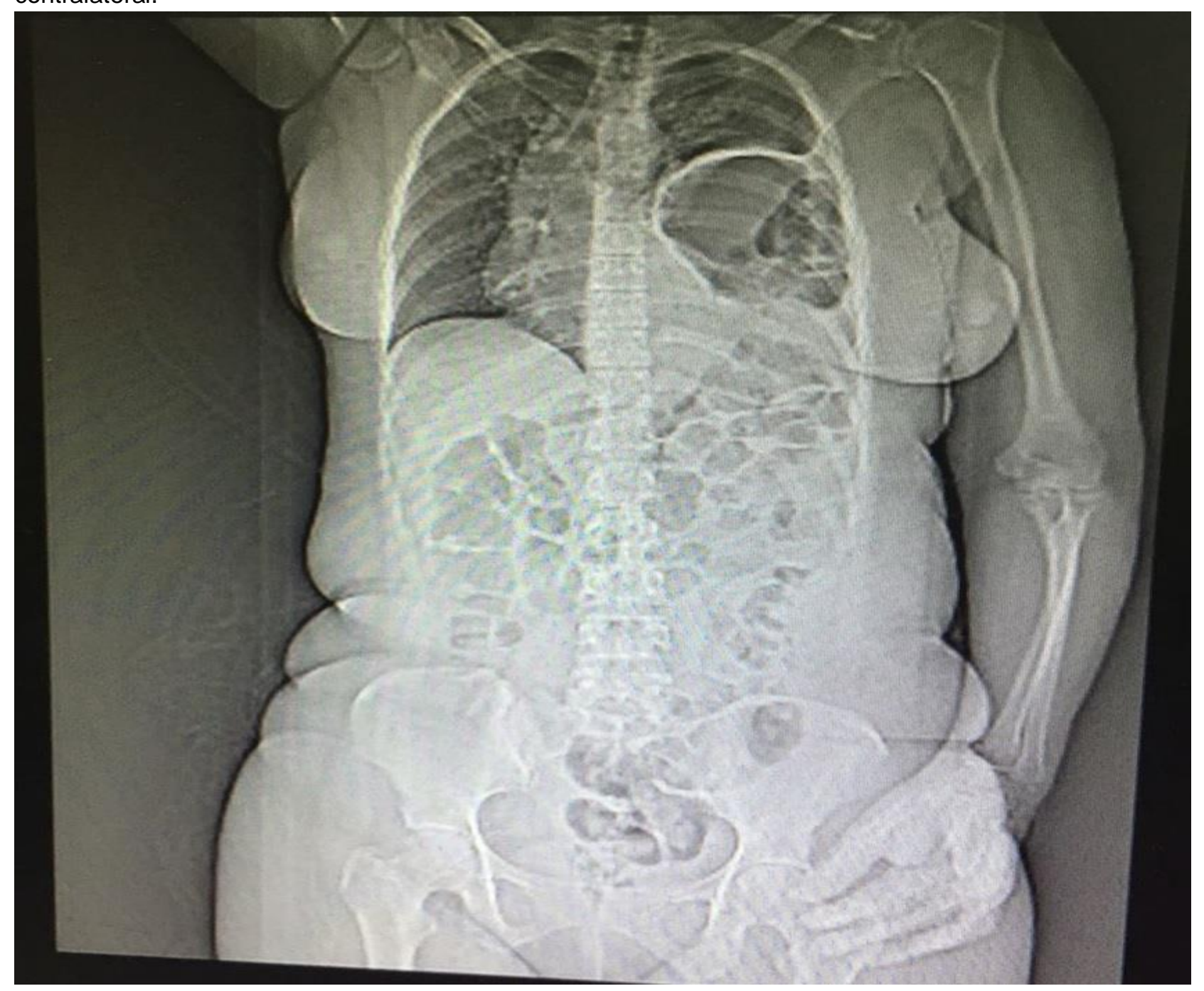

Fonte: Dados da pesquisa, 2018.

Paciente foi submetida a cirurgia exploradora, com acesso por laparotomia mediana supra e infra umbilical. Durante a inspeção da cavidade foi visualizada um defeito de aproximadamente 16 centímetros na hemicúpula diafragmática esquerda, com grande quantidade de vísceras abdominais. A lise de estruturas aderidas foi realizada em região de transição toracoabdominal e seguiu-se com a redução das vísceras para retorno e reposicionamento em cavidade abdominal, sendo que está apresentava em seu conteúdo estômago, flexura cólica esquerda com grande parte de cólon transverso e descendente, alças de jejuno, baço e lobo esquerdo do fígado em cavidade torácica. A lesão em diafragma foi corrigida em suturas contínua com polipropileno monofilamentar em chuleio simples em apenas um plano, seguido de drenagem fechada de tórax (DFT) à esquerda.

Ao pós-operatório imediato, a paciente teve uma boa recuperação em pós-anestésico, com drenagem regular de líquido em frasco de DFT. Não apresentou queixas abdominais como parada de evacuação ou dor abdominal nos dias de internação seguintes. No décimo dia de pós-operatório, já sem drenagem de quantidades elevadas de fluidos, foi realizada a alta hospitalar da paciente já com a retirada do dreno torácico e sem queixas de dor no local da ferida operatória. 


\section{DISCUSSÃO}

A relação entre a quantidade de material intratorácico em hérnias diafragmáticas não está relacionada diretamente com os sintomas apresentados, e mesmo na literatura atual, não existem relações desta natureza. Isso nos leva a criar uma cautela extra ao examinar pacientes que estão em períodos pós-trauma, principalmente contusos. Estes mesmos pacientes poderiam apresentar uma lesão mínima no momento do trauma que aumentou com o tempo, ou semelhantes a este caso, com uma grande quantidade de vísceras abdominais em cavidade torácica, mas com poucas repercussões sistêmicas. (BLITZ M e LOUIE BE, 2009).

O principal exame complementar em questão de especificidade e sensibilidade é a tomografia computadorizada de tórax e abdome, superando a radiografia devido à melhor visualização das alças intestinais melhorando o planejamento cirúrgico para o caso. Especificamente, o exame radiográfico pode ser utilizado como primeira linha na pesquisa diagnóstica, já que na maioria dos casos, há sinal de elevação de nível de bulha gástrica, principal sinal na presença de hérnia diafragmática, muito sugestivo quando associado a clínica clássica de dispneia, dor em andar superior do abdômen e murmúrio vesicular diminuído em hemitórax ipsilateral à lesão. Portanto não devemos excluir a radiografia, pois além da sua praticidade, esta é mais barata e mais acessível a unidades de saúde (ATIF Q e KHALID T, 2016).

A tomografia computadorizada faz-se necessária quando há duvidas no diagnóstico. Este cenário se mostra presente quando a clínica do paciente não é sugestiva da lesão, ou até, quando não temos história de traumas passados de relevância para o paciente. A exemplo, hérnias congênitas necessitam da avaliação tomográfica. Existem duas situações menos presentes na literatura que apresentam melhor avaliação com este exame, que são na presença de lesão mínima do músculo, mas com clínica sugestiva de hérnia, e quando a lesão está em fase inicial. Devido ao contexto do primeiro atendimento e a provável falta de sinais clínicos, o diagnóstico de lesão diafragmática em estágio inicial é raríssimo, podendo até ser subdiagnosticado durante análise de exames de imagem. Laparotomia exploradora ou laparoscopia podem elucidar casos duvidosos também, mas são muito invasivos para elucidação diagnóstica inicial (BLITZ M e LOUIE BE, 2009).

A questão discutida recentemente é a realização de reparo cirúrgico por acesso abdominal, torácico ou por ambos. Não existe consenso atual em qual o melhor método, depende da equipe cirúrgica responsável, de seu treinamento prático e de fatores estruturais da instituição, nem existem trabalhos que evidenciem resultados melhores nos pós-operatórios em determinado acesso, até porque há diversas variáveis nos casos estudados (SILVA GP, et al., 2018). A laparotomia é mais utilizada nos relatos agudos e a toracotomia em tardios, devido à presença de aderências (ATIF Q e KHALID T, 2016). Também não há grandes discussões sobre o reparo cirúrgico da lesão, tanto o tipo de sutura quanto da necessidade ou não de suporte com tela de polipropileno.

Outro fator diferente deste caso somado a pouca quantidade de características clínicas, é a ausência de complicações relevantes durante o pós-operatório. A complicação mais comum é a pneumonia devido a presença de alças intestinais previamente, a paciente pode até já chegar com quadro de pneumonia dependendo do tempo. No entanto, obstrução intestinal, isquemia vascular e falência renal estão relacionadas com esta doença (ABDELSHAFY M e KHALIFA YSE, 2018).

\section{CONSIDERAÇÕES FINAIS}

As lesões diafragmáticas podem ser diagnosticadas imediatamente, em meses ou anos após o trauma. Este relato ilustra mais um caso raro de hérnia diafragmática traumática pouco sintomática em contraste com o grande volume de alças intestinais em cavidade torácica, mas que poderia ser suspeitado pela história previa de trauma de maneira tranquila, assim como a discreta alteração na ausculta pulmonar, e com a realização de exame adequado (KUMAR S, et al., 2018). Entretanto, são estes os detalhes mais relevantes que podem passar despercebido por alguns profissionais, seja por descuidos ou cansaço da rotina de plantões, onde estes pacientes são atendidos na maior parte no nosso sistema atual de saúde. Trazendo a nossa região, o Amazonas apresenta regiões distantes no interior com apoio médico dificultado e poucos centros de referência, o que dificulta a mobilidade destes pacientes em caso de queixas menores.

REAS/EJCH | Vol. Sup. 22 | e663 | DOI: https://doi.org/10.25248/reas.e663.2019 Página 5 de 6 


\section{REFERÊNCIAS}

1. ABDELSHAFY M, KHALIFA YSE. Traumatic diaphragmatic hernia challenging diagnosis and early management. J Egypt Soc Cardio-Thoracic Surg [Internet]. Elsevier Ltd; 2018;26(3):219-27. Available from: https://doi.org/10.1016/j.jescts.2018.07.001

2. ALLAN Z, et al. Traumatic diaphragmatic rupture with underlying lung laceration and tension pneumoperitoneum. J Surg Case Reports. 2017;6:1-3.

3. ATIF Q, KHALIQ T. Traumatic Right Diaphragmatic Hernia; A Delayed Presentation. J Ayub Med Coll Abbottabad. 2016;28(3):625-6.

4. BLITZ M E LOUIE BE. Chronic Traumatic Diaphragmatic Hernia. Thorac Surg Clin NA [Internet]. Elsevier Ltd; 2009;19(4):491-500. Available from:http://dx.doi.org/10.1016/j.thorsurg.2009.08.001

5. ČALA Z, et al. Laparoscopic Treatment Of Morgagni Hernia: Report Of Three Cases. Acta Clin Croat. 2017;56(2):318-22.

6. ERCAN M, et al. Dual mesh repair for a large diaphragmatic hernia defect : An unusual case report. Int J Surg Case Rep. 2016;28(6):266-9.

7. JÚNIOR GAP. Hérnia diafragmática traumática. Rev Col Bras Cir. 2000;375-82.

8. KUMAR S, et al. An undiagnosed left sided traumatic diaphragmatic hernia presenting as small intestinal strangulation: A case report. Int J Surg Case Rep [Internet]. Surgical Associates Ltd; 2013;4(5):446-8. Available from: http://dx.doi.org/10.1016/j.ijscr.2013.02.006

9. MOORE EE, et al. Organ injury scaling. Surg Clin North Am [Internet]. Elsevier Masson SAS; 1995;75(2):293-303. Available from: http://dx.doi.org/10.1016/S0039-6109(16)46589-8

10. SILVA GP, et al. Thoracotomy compared to laparotomy in the traumatic diaphragmatic hernia . Systematic review and proportional. Acta Cir Bras. 2018;33(1):49-66.

11. ZHAO H, et al. Delayed traumatic parasternal hernia causes jejunal necrosis: A case report. Trauma Case Reports [Internet]. Department of Thoracic Surgery, No. 1 Hospital of BaoDing City ,China; 2017;9(1):30-3. Available from: http://dx.doi.org/10.1016/j.tcr.2017.05.006. 